\title{
Mitotic control of human papillomavirus genome-containing cells is regulated by the function of the PDZ-binding motif of the E6 oncoprotein
}

\author{
Elizabeth K. Marsh ${ }^{1,4}$, Craig P. Delury ${ }^{1}$, Nicholas J. Davies ${ }^{1}$, Christopher J. Weston ${ }^{2}$, \\ Mohammed A. L. Miah ${ }^{1}$, Lawrence Banks ${ }^{3}$, Joanna L. Parish ${ }^{1}$, Martin R. Higgs ${ }^{1, *}$, \\ Sally Roberts ${ }^{1, *}$ \\ ${ }^{1}$ Institute of Cancer and Genomic Sciences, University of Birmingham, Birmingham, United Kingdom \\ ${ }^{2}$ Institute of Immunology and Immunotherapy, University of Birmingham, Birmingham, United Kingdom \\ ${ }^{3}$ International Centre for Genetic Engineering and Biotechnology, Padriciano, Trieste, Italy \\ ${ }^{4}$ Present address: Faculty of Medicine, Dentistry and Health, University of Sheffield, Sheffield, United Kingdom \\ * These authors have contributed equally to this work \\ Correspondence to: Sally Roberts, email: s.roberts@bham.ac.uk \\ Keywords: human papillomavirus, E6, PDZ proteins, HPV life cycle, mitosis \\ Received: August 26, $2016 \quad$ Accepted: December 18, $2016 \quad$ Published: January 03, 2017
}

\section{ABSTRACT}

The function of a conserved PDS95/DLG1/ZO1 (PDZ) binding motif (E6 PBM) at the C-termini of E6 oncoproteins of high-risk human papillomavirus (HPV) types contributes to the development of HPV-associated malignancies. Here, using a primary human keratinocyte-based model of the high-risk HPV18 life cycle, we identify a novel link between the E6 PBM and mitotic stability. In cultures containing a mutant genome in which the E6 PBM was deleted there was an increase in the frequency of abnormal mitoses, including multinucleation, compared to cells harboring the wild type HPV18 genome. The loss of the E6 PBM was associated with a significant increase in the frequency of mitotic spindle defects associated with anaphase and telophase. Furthermore, cells carrying this mutant genome had increased chromosome segregation defects and they also exhibited greater levels of genomic instability, as shown by an elevated level of centromere-positive micronuclei. In wild type HPV18 genome-containing organotypic cultures, the majority of mitotic cells reside in the suprabasal layers, in keeping with the hyperplastic morphology of the structures. However, in mutant genome-containing structures a greater proportion of mitotic cells were retained in the basal layer, which were often of undefined polarity, thus correlating with their reduced thickness. We conclude that the ability of E6 to target cellular PDZ proteins plays a critical role in maintaining mitotic stability of HPV infected cells, ensuring stable episome persistence and vegetative amplification.

\section{INTRODUCTION}

Of the more than 200 human papillomavirus (HPV) types that are known to infect humans, twelve have been defined as human carcinogens (HPV16, 18, 31, 33, 35, $39,45,51,52,56,58$ and 59), with another twelve types that are closely related to the carcinogenic viruses, defined as "probably" or "possibly carcinogenic" [1]. These socalled high-risk (HR) viruses are closely associated with the development of epithelial cancers of the anogenital and oropharyngeal tracts [2-4]. The continuous expression of the HPV early proteins E6 and E7 is one of the defining features of HPV driven carcinogenesis and thus, they are attractive targets for therapeutic intervention of these tumours. The ability of E6 and E7 proteins to deregulate host mechanisms of cell cycle control, senescence and survival can lead to cellular immortalization, and the development of genetically unstable cells that are at increased risk of oncogenic transformation [5]. The key biological activities of the E6 oncoprotein that contribute to carcinogenesis include the capacity to inactivate the p53 tumor suppressor protein, activation of telomerase 
and the targeting of specific cellular proteins containing PDZ (SD95/ㅁLG1/Z्ZO-1) domains (referred to as PDZ proteins) [6].

Binding between PDZ proteins and E6 is mediated by a C-terminal class I PDZ domain-binding motif (PBM) that is only found in E6 proteins of the HR-HPV types, suggesting that the motif acts as an oncogenic signature. Indeed, the E6 PBM was shown to be necessary for the morphological transformation and the induction of tumorigenesis of rodent cell lines [7]. Moreover, in a transgenic mouse model of cervical carcinogenesis, the presence of the HPV16 E6 PBM function enhanced tumor development, affecting both tumor size and area of tumor invasion [8]. In mammalian keratinocytes, the E6 motif has been linked to cell proliferation $[9,10]$ and whilst not necessary for the immortalization of these cells, it has been shown to contribute to epithelial-mesenchymal transition and anchorage-independent cell growth; two acquired phenotypes that are linked to invasive and metastatic growth of tumors [11-14].

The interaction between E6 and PDZ proteins most often leads to ubiquitin-mediated degradation of the host protein via the proteasome [15], although this is not the case for all PDZ targets [16, 17]. Phosphorylation of the E6 PBM has been shown to negatively regulate the interaction with PDZ protein partners and confers the ability of the E6 protein to interact with a number of 14-3-3 isoforms $[18,19]$. Many of the PDZ proteins that associate with E6 have roles in governing cell polarity, including apico-basal epithelial polarity and asymmetric cell division. In fact, core components of all the apico-basal polarity modules (Crumbs, Par and Scribble) are targets of the E6 domain. These include PALS1-associated tight junction protein (PATJ) of the Crumbs complex [20], PDZ portioning defective 3 protein (PAR3) of the Par complex [16] and the human homologues of the Drosophila tumor suppressor proteins, discs large 1 (DLG1) and scribble (hSCRIB), both of the Scribble complex [7, 15, 21, 22]. Other PDZ targets of E6 include members of the MAGI family of proteins, which are also linked to polarity $[23,24]$, and the non-receptor tyrosine phosphatases PTPN3 and PTPN13, that both have roles in regulating signal transduction pathways involved in cell proliferation, apoptosis, migration and intracellular trafficking $[25,26]$.

The function of the E6 PBM has been shown to play an essential role in the replication cycle of HR-HPV types. Our studies using organotypic raft cultures of primary human foreskin keratinocytes (HFK) transfected with a mutant HPV18 genome which expresses an E6 protein lacking the PBM (E6 $\triangle \mathrm{PDZ}$ ), showed a deleterious effect of loss of this E6 function upon the productive phase (viral episome amplification and viral late gene expression) of the virus life cycle [27]. Further analysis of these cultures demonstrated that the defect in the productive cycle was linked to a reduction in cell proliferation in the upper layers of the stratified cultures. Both viral episome establishment and stable maintenance of the viral episomes were also compromised upon monolayer cell growth of the basal-like undifferentiated cells [27], observations that were supported by investigations of other HR-HPV types $[28,29]$. Collectively, these studies indicate that the E6 PBM is critical for multiple stages of the infectious cycle of cancer-causing HPV types.

In this study, we have investigated in greater detail the impact of loss of the E6 PBM function on the growth of viral genome-containing keratinocytes. We show that this function of E6 is strongly associated with the maintenance of mitotic stability of episome-containing cells. In the absence of the E6 PBM, cells develop severe mitotic abnormalities and accrue genomic instability. Thus, this function of E6 is vital for episome maintenance and vegetative replication, potentially by acting to safeguard mitotic integrity of HPV-infected cells.

\section{RESULTS}

As described previously, we genetically engineered the wild type (WT) HPV18 genome to replace codons at positions 155 and 156 of the E6 protein with translation termination codons, creating the mutant genome E6 $\triangle \mathrm{PDZ}$ [27]. Thus, the E6 protein expressed from the mutant genome lacked the $\mathrm{C}$-terminal four amino acids constituting the PBM $\left({ }^{155} \mathrm{ETQV}^{158}\right)$. The deletion of the HPV18 E6 PBM negates the targeting of PDZ proteins but does not interfere with other E6 functions, including the ability of the E6 protein to degrade the p53 tumor suppressor protein [27]. We established HPV18 genome-containing cell lines by transfecting primary human foreskin keratinocytes (HFK) from three different donors (\#1, \#2 and \#3) with the WT genome or the mutant genome. Both the WT and E6 $\Delta \mathrm{PDZ}$ genomes replicated as extrachromosomal plasmids in these cells (Figure 1A); although E6 $\triangle \mathrm{PDZ}$ was present at reduced copy number in comparison to the WT episomes, in agreement with our previous findings [27]. Notably, the E6 $\triangle \mathrm{PDZ}$ mutation does not adversely affect the levels of the E6, or the E7 proteins expressed in the HPV18 genomecontaining cells grown in undifferentiated monolayer culture or in organotypic raft culture [27].

Since extended cell culture of E6 $\triangle \mathrm{PDZ}$ cell lines often leads to a sharp reduction in maintenance of episomes and viral DNA integration [27], only cell passages ( $\leq 35$ cell population doublings) prior to these viral genetic events (as determined by Southern analysis) were used in this study.

\section{Deletion of the E6 PBM is associated with enhanced frequency of nuclear atypia of HPV18 genome-containing cells}

By using bright-field microscopy, it was noticeable that in monolayer cell growth conditions, in comparison to the untransfected donor HFK, both the WT and E6 $\triangle \mathrm{PDZ}$ 
genome-containing cell cultures were characterized by the presence of cells with multiple, and often misshapen nuclei (Figure 1B). Quantification of HFK cells showed that $0.43 \%$ of cells had this aberrant multinucleated morphology ( $\geq 3$ nuclei/cell), whereas the percentage of multinucleated cells increased by 7 -fold in WT genome containing cells (3.15\%) (Figure 1C). Interestingly, multinucleation of cells harboring the E6 $\triangle \mathrm{PDZ}$ genomes was enhanced further (17-fold change; $7.47 \%$ of cells) (Figure 1C). Furthermore, cells containing the E6 $\Delta \mathrm{PDZ}$ genomes showed a 2-fold increase in frequency of cells with high levels of multinucleation ( $\geq 5$ nuclei/cell) in comparison to those carrying the WT genomes, increasing from $1.61 \%$ (WT) to $3.26 \%$ (E6 $\triangle \mathrm{PDZ}$ ). These data demonstrate that the replication of HR-HPV episomes in primary keratinocytes leads to perturbation of cell division, in agreement with previous suggestions [30-32]. Moreover, our analysis also suggests that loss of the E6 PDZ binding function further increases the deleterious effect of the virus on coordinated cell division.

\section{Live cell microscopy reveals an increase in frequency of cells undergoing abnormal mitosis in $\mathrm{E} 6 \Delta \mathrm{PDZ}$ cell cultures}

To investigate whether the increase in multinucleated cells observed upon loss of the E6 PBM was due to increased mitotic errors, we next examined mitotic progression of the cells by live cell imaging of asynchronous monolayer cell cultures. Cells carrying either WT or E6 $\triangle \mathrm{PDZ}$ genomes were plated at low cell density and once individual cell colonies were visible they were imaged for $16 \mathrm{~h}$ by phase contrast microscopy, using the Cell-IQ system. The resulting time-lapse movies were subsequently analyzed to determine the number of mitotic events within individual colonies, and each mitotic event classified as 'normal' or 'abnormal' according to defined criteria (e.g. time taken from mitotic entry to daughter cell separation, mitotic spindle polarity, visible and equal separation of condensed chromosomes). Images of representative examples of normal and abnormal mitotic

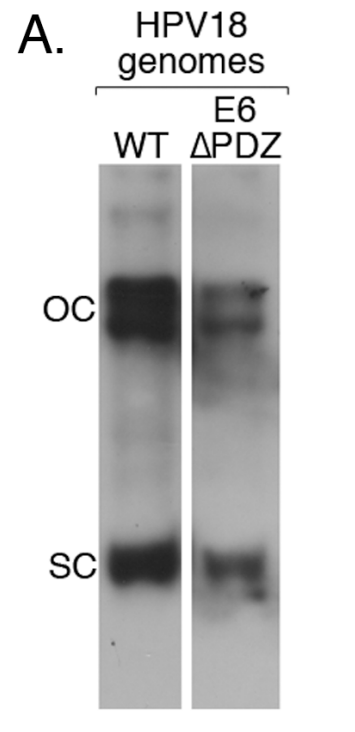

B. HPV18 genomes

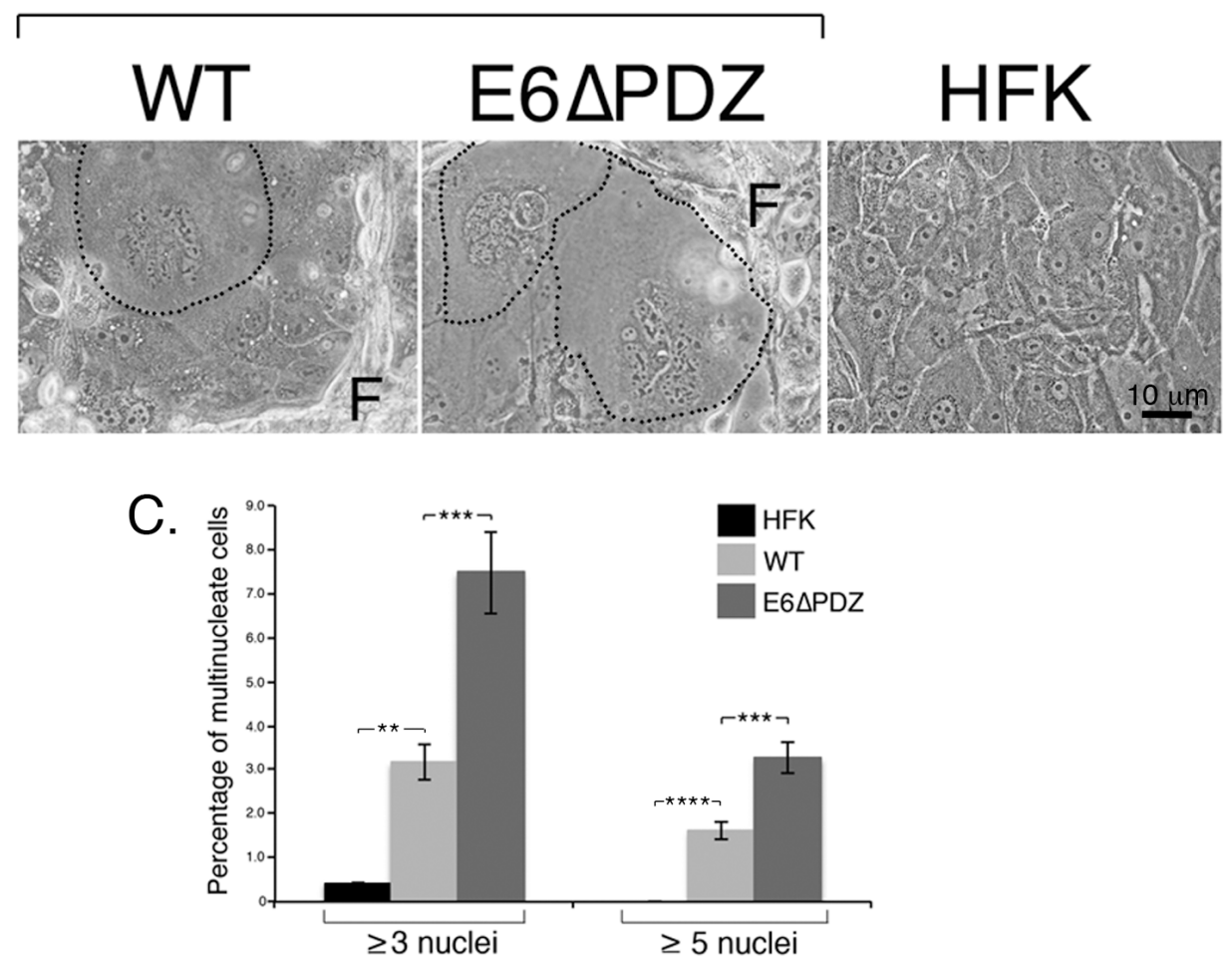

Figure 1: Loss of E6 PBM function is associated with enhanced frequency of nuclear atypia of viral genome-containing cells. A. Southern analysis confirmed presence of HPV18 wild type (WT) and mutant (E6 $\triangle \mathrm{PDZ}$ ) episomes migrating as supercoils (SC) and open circles (OC) in cells; the mutant genomes establish at a lower copy number than the WT genomes as shown previously (the images of the two lanes were taken from the same Southern blot at a single exposure) [27]. B. Phase contrast images of cells grown in monolayer culture, including untransfected donor cells (HFK), with fibroblast feeder cells (F). HPV18 genome-containing cells showing nuclear atypia (multinucleation) are identified (black dotted line). C. Bar graph, percentage of cells containing multiple nuclei; data (mean \pm standard error of the mean) derived from random fields of view and cells from three independent donors. Student $t$ test was used to determine the significance (**, $\mathrm{P}<0.01$; ***, $\mathrm{P}<0.001 ; * * * *, \mathrm{P}<0.0001)$. Total number of cells counted: HFK, 2000 cells; WT, 1400 cells; E6 $\Delta \mathrm{PDZ}, 1060$ cells. 
cell divisions are given in Figure 2A and 2B respectively, and movies are shown in Supplementary Figure 1. In order to eliminate any donor keratinocyte effects, the analysis was performed on cell lines established in the three different keratinocyte backgrounds.

The distributions of normal and abnormal mitoses in the HPV18 genome-containing cells are shown in Figure $2 \mathrm{C}$. The majority of mitotic cells carrying the WT genomes underwent normal cell division, with less than $25 \%$ (a range of $14.5 \%$ to $24.6 \%$ between the three donors) undergoing an aberrant division. However, in cells carrying the E6 $\triangle \mathrm{PDZ}$ genomes, the proportion of mitotic cells undergoing abnormal cell division increased markedly across all three donors (a range of $45.5 \%$ to $60.8 \%$ ). Moreover, we also noted that in E6 $\triangle \mathrm{PDZ}$ containing cell lines, even cells with elevated numbers of nuclei $(>15)$ entered mitotic division (Supplementary Figure 2). In the examples given in Figure 2A, the time to complete a normal bipolar mitotic division for both WT and E6 $\triangle \mathrm{PDZ}$ genome-containing cells is just over $1 \mathrm{~h}$, with no noticeable differences in the time taken to complete mitosis between cell lines. However, the abnormal WT and E6 $\triangle \mathrm{PDZ}$ divisions are multi-radial and time to completion is extended to over $1 \mathrm{~h} 45 \mathrm{~min}$ (Figure 2B). Taken together, these data indicate that in the context of whole HR-HPV genomes, loss of E6 PBM function is associated with an increase in the frequency of abnormal mitotic events.

\section{Loss of E6 PBM function is associated with an increase in spindle-associated abnormalities in late stage mitosis}

Next, we wanted to further characterize the mitotic defect observed in the E6 $\triangle \mathrm{PDZ}$ genome-containing cells. Therefore, cells were grown in monolayer cell culture on glass coverslips, fixed and stained for the mitotic spindle protein alpha-tubulin and counterstained with the DNA dye DAPI. As before, mitotic cells were scored from random fields of view as to whether 'normal' or 'abnormal'; the specific events were chromosome alignment on spindle and spindle polarity in metaphase; successful separation of chromosomes, spindle polarity in anaphase and telophase, the presence of anaphase or telophase bridges, and telophase spindle polarity.

Both WT and E6 $\mathrm{PDDZ}$ cells were identified in prophase, metaphase, anaphase and telophase in all three individual donor backgrounds. In agreement with the findings from the live microscopy analysis (Figure 2 ), increased abnormal mitoses were observed in cells harboring the mutant E6 $\triangle \mathrm{PDZ}$ genomes (data not shown). Unlike the normal mitotic cells, which were mainly in anaphase-telophase, the majority of abnormal mitotic WT and E6 $\triangle \mathrm{PDZ}$ genome-containing cells were in metaphase (Figure 3A). Moreover, whilst very few of the cells carrying WT genomes had defects in anaphase and telophase, in comparison, the proportion of E6 $\triangle \mathrm{PDZ}$ cells with spindle abnormalities in both anaphase and telophase increased in all three donors (Figure 3A, i, and $3 B)$. Thus, in donor \#1, abnormalities in anaphase and telophase of E6 $\triangle \mathrm{PDZ}$ genome-containing cells increase by 2.7 -fold, in donor \#2 by 5.6 -fold and in donor \#3 by 3.0-fold, compared to cells harboring the WT genomes. These data suggest that whilst only a small proportion of cells harboring WT HPV18 genomes with metaphase abnormalities are able to progress through the mitotic cycle, a greater proportion of abnormal cells containing the mutant genome are able to move into anaphase and telophase. Examples of mitotic abnormalities detected in the HPV18 genome-containing cells are shown in Figure 3B.

To further define the underlying defects occurring in anaphase and telophase in the E6 $\triangle \mathrm{PDZ}$ cells, we next analyzed the frequency of specific mitotic abnormalities in anaphase (anaphase bridges, lagging chromosomes, spindle polarity) and telophase (spindle polarity, telophase bridge) (see Figure 3B for examples of these defects in E6 $\triangle$ PDZ cells). From this analysis, it was shown that there was increased frequency of defects associated with anaphase and telophase in E6 $\triangle \mathrm{PDZ}$ genome-containing cells compared to the WT genome controls (Figure 3C). Indeed, the combined frequency of these abnormalities was statistically significant $(\mathrm{p}<0.05)$ relative to the cells harboring the WT genomes, although the difference in the frequency of any one abnormality did not reach statistical significance (Figure 3C and 3D). Together, these data suggest that loss of the E6 PBM function is linked to a spindle abnormality in the viral episome-containing cells and not a signaling defect associated with one specific mitotic abnormality. In conclusion, in HPV18 genomecontaining cells, the loss of E6 PDZ binding resulted in a quantitative increase in mitotic defects persisting late into mitosis.

\section{Increased frequency of centromere-positive micronuclei in E6 $\triangle \mathrm{PDZ}$ genome-containing cells}

Our findings that loss of the E6 PBM function led to an increase in the frequency of spindle abnormalities in anaphase and telophase suggested that cells harboring the E6 $\triangle \mathrm{PDZ}$ genomes would exhibit increased chromosome segregation defects. One consequence of these is the partition of chromosomes into micronuclei, whereby nuclear membranes form around lagging chromosomes and are visible in interphase cells as discrete extranuclear formations of enveloped DNA. Therefore, to investigate this possibility, the frequency of cells with micronuclei was determined in cells harboring the HPV18 genomes (Figure 4A). Micronuclei were observed in cells replicating either WT or E6 $\triangle \mathrm{PDZ}$ genomes, but the frequency of cells with micronuclei increased in the presence of E6 $\triangle \mathrm{PDZ}$ genomes $(1.3$ - 2.9 fold increase 

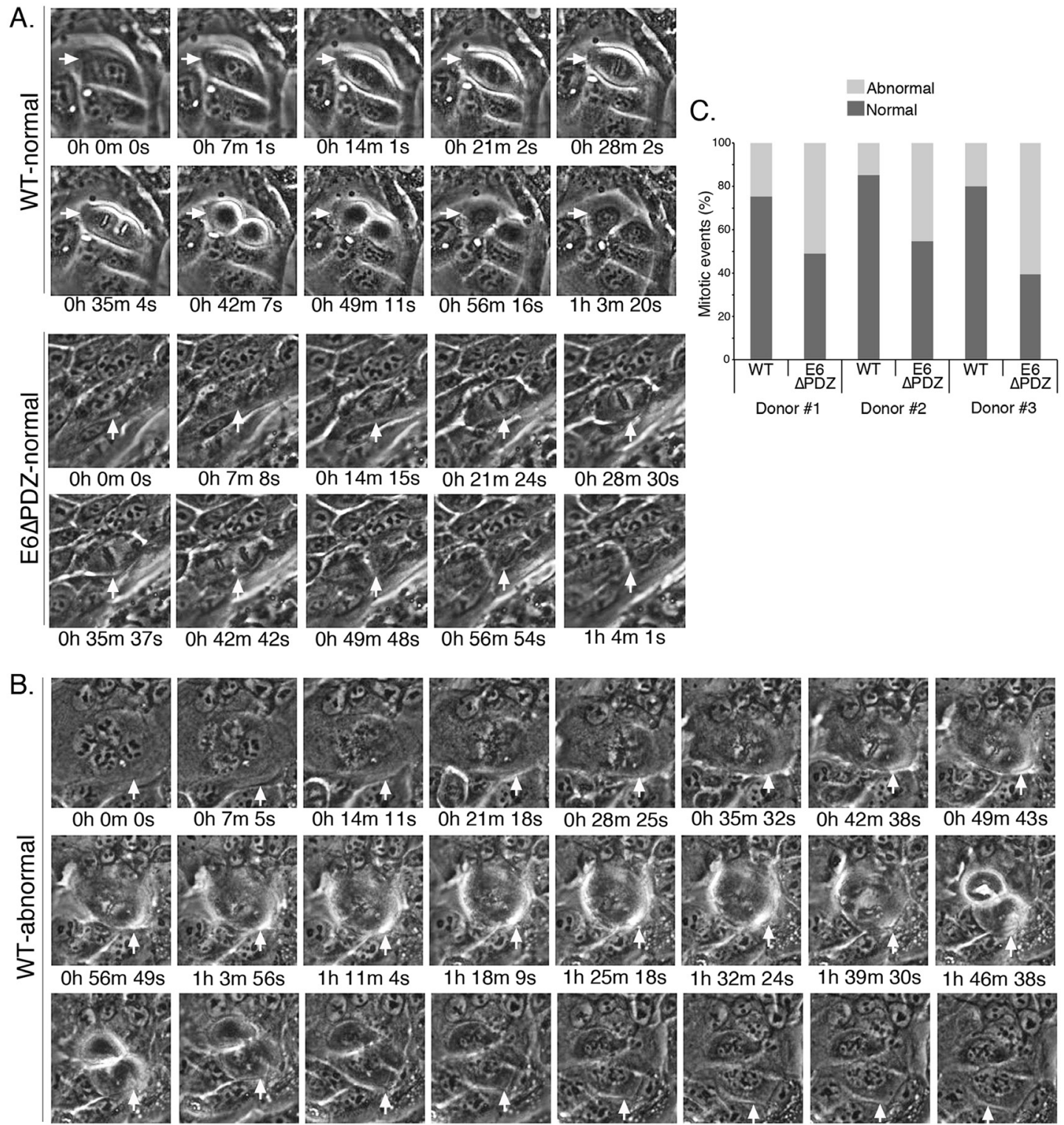

Oh $42 \mathrm{~m} 38 \mathrm{~s}$ Oh $49 \mathrm{~m} 43 \mathrm{~s}$

$1 \mathrm{~h} 3 \mathrm{~m} 56 \mathrm{~s}$

$1 \mathrm{~h} 11 \mathrm{~m} \mathrm{4s}$

1h $18 \mathrm{~m} 9 \mathrm{~s}$

$1 \mathrm{~h} 25 \mathrm{~m} \mathrm{18s}$

$1 \mathrm{~h} 32 \mathrm{~m} 24 \mathrm{~s}$
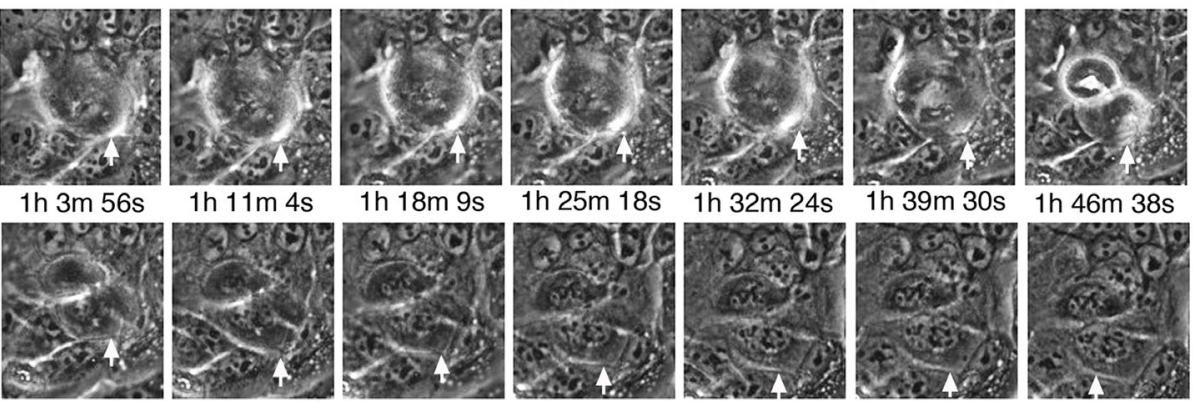

1h $46 \mathrm{~m} 38 \mathrm{~s}$
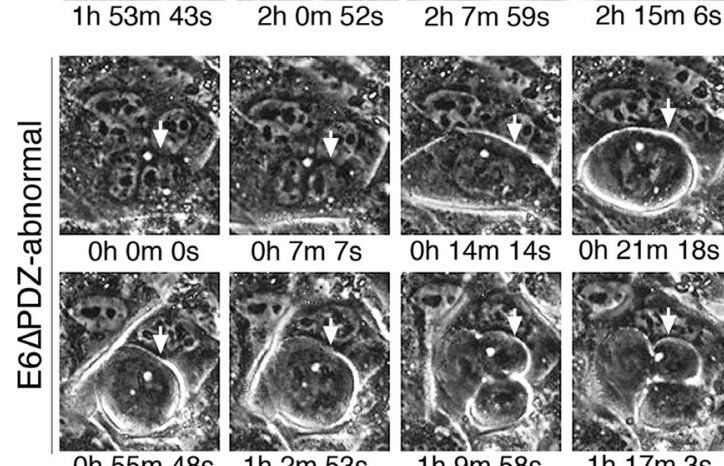

$2 \mathrm{~h} 22 \mathrm{~m} \mathrm{14s}$
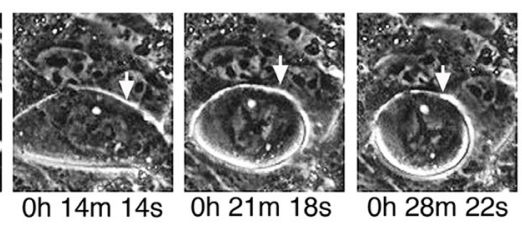

2h 29m 21s

$2 \mathrm{~h} 36 \mathrm{~m} \mathrm{27s}$
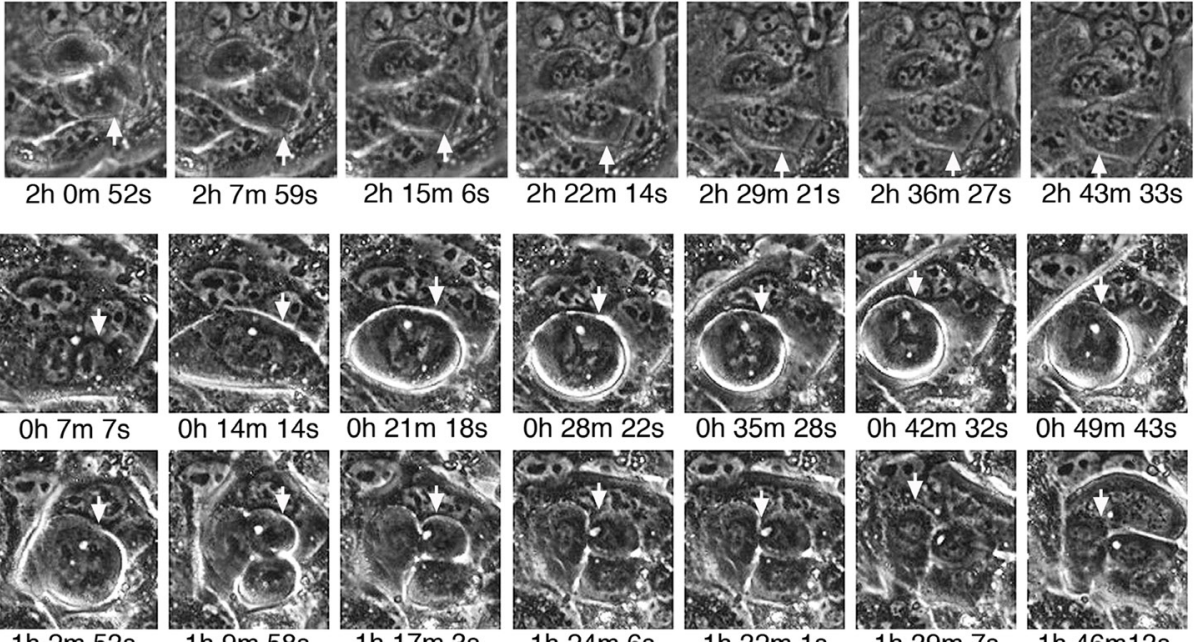

$1 \mathrm{~h} 24 \mathrm{~m} 6 \mathrm{~s}$

$1 \mathrm{~h} 32 \mathrm{~m} 1 \mathrm{~s}$

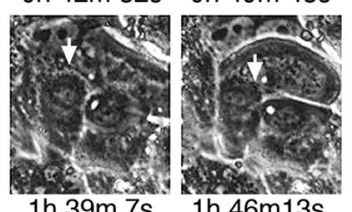

Figure 2: Live cell microscopy shows that loss of E6 PBM function is associated with an increase in abnormal mitoses in viral genome-containing cells. Examples of normal A. and abnormal B. mitoses from the live cell microscopy in cell lines harboring the WT or the E6 $\triangle \mathrm{PDZ}$ genomes (movies given in Supplementary Figure 1). Cells undergoing mitosis are identified with a white arrow and the time to complete mitosis is given. Note that in these examples of abnormal mitosis, the event is multipolar and the time taken to completion is much longer than for completion of normal cell division. C. Bar graph, percentage of mitoses that were normal or abnormal in each of the three donor backgrounds. Mitoses defined as normal if bipolar, had visible and equal separation of condensed chromatin and the time to completion of mitosis was not excessive; otherwise the mitotic event was scored as abnormal. Number of colonies counted was in the range of 14 to 23 and for each of the cell lines between 90-308 mitoses counted. 
compared to WT genome-containing cells), although these increases only reached significance in two out of the three donors analyzed. These data therefore support our previous conclusion that the loss the E6 PBM function enhances the dysfunction of the mitotic apparatus.

Since micronuclei formation occurred in the presence of WT as well as the mutant genomes, it was important to establish if they arose through similar mechanisms. Micronuclei may arise from both chromosome segregation errors to give centromerepositive micronuclei; or through failed separation of chromosome fragments to produce centromere-negative micronuclei. Therefore, cells were stained with an antibody that detects the centromere protein, centromere protein A (CENPA) and micronuclei were then scored as CENPA-positive or -negative (Figure 4B). In both
A.

(i)

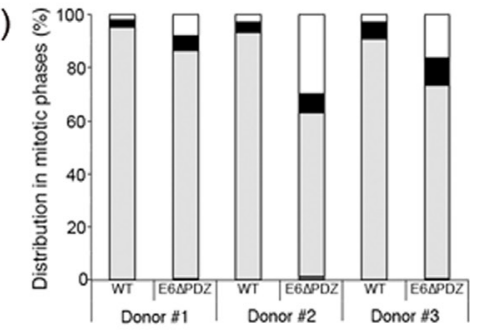

ABNORMAL

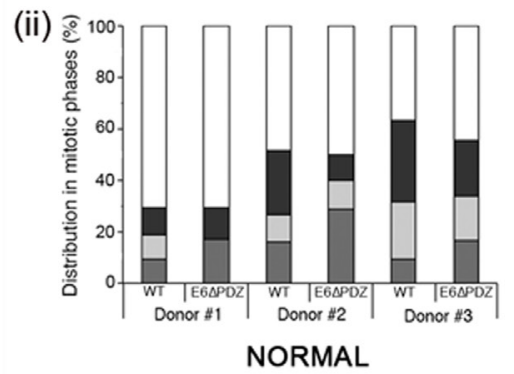

C.

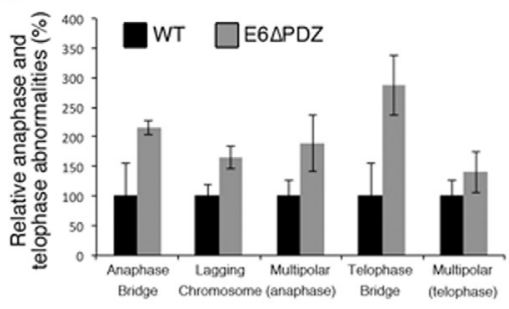

D.

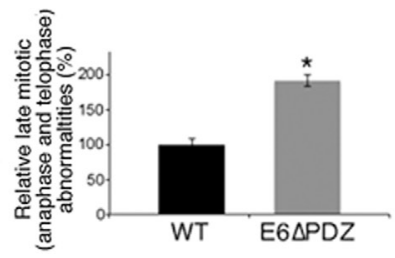

B. $\alpha$-tubulin
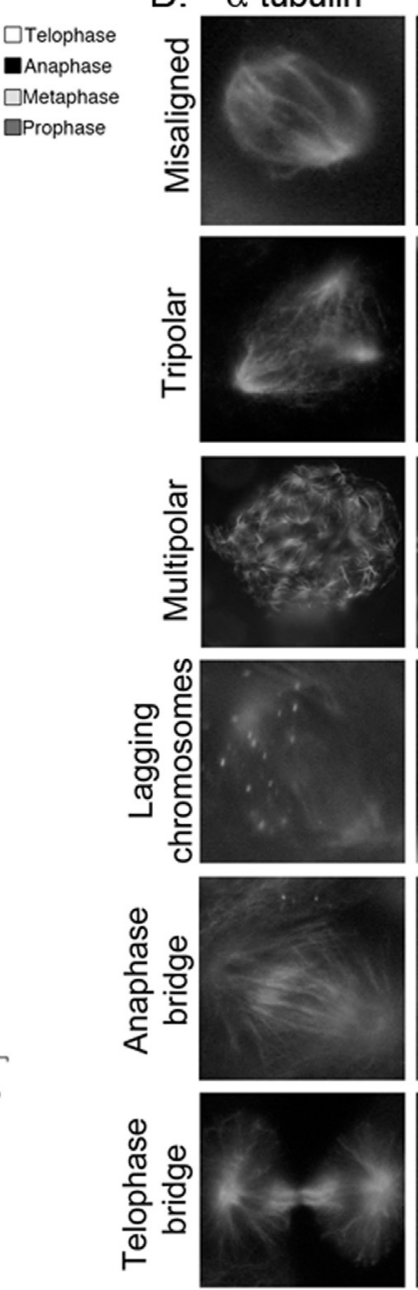
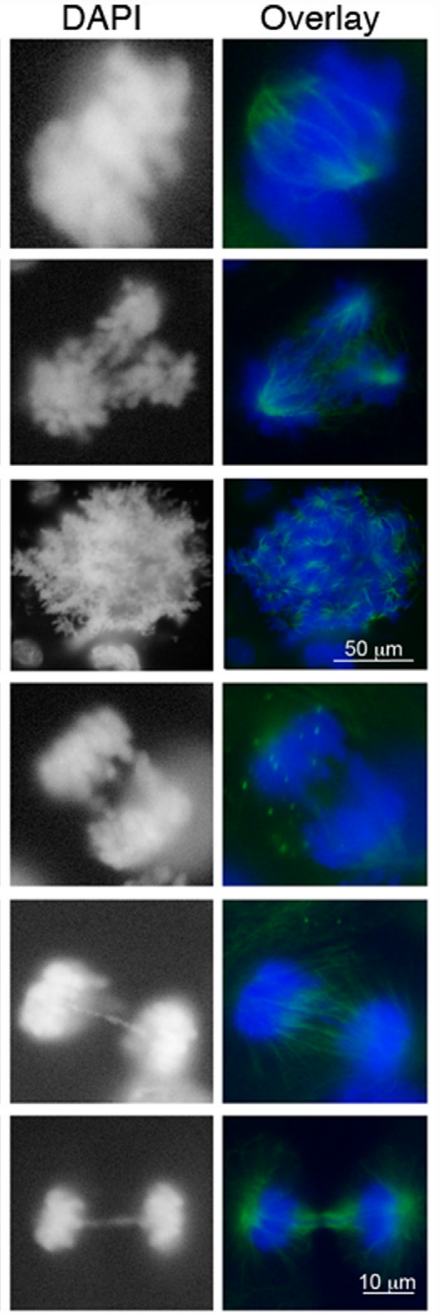

Figure 3: Loss of E6 PBM function leads to an enhancement in the frequency of abnormal cells progressing into anaphase and telophase. A. Abnormal (bar graph i) or normal (bar graph ii) mitoses were scored in each stage of mitosis (prophase, metaphase, anaphase and telophase) by $\alpha$-tubulin staining and counterstaining with DAPI. The data are derived from three independent experiments for each of the three donors and presented as a proportion of total abnormal (top graph) or normal (bottom graph) mitoses. For each experiment, a minimum of 200 cells per donor was counted. B. Examples of abnormal mitoses in HPV18 genome-containing cells showing spindle morphology ( $\alpha$-tubulin, green) and chromosomes (DAPI, blue). C. Individual mitotic abnormalities were scored by $\alpha$-tubulin staining and counterstaining with DAPI. Only the data for anaphase and telophase abnormalities are shown. The data are derived from three independent experiments for each of the three donors, with a minimum of 100 abnormal mitotic cells scored for each experiment, and the data is shown as the mean ( \pm standard error of the mean). The differences in the frequency of individual abnormalities between WT and E6 $\triangle \mathrm{PDZ}$ cells were not statistically significant. D. The frequency of the combined late (anaphase and telophase) mitotic abnormalities in the E6 $\triangle \mathrm{PDZ}$ genome containing cells shown relative to cells harboring the WT genomes. Student's $t$ test was used to determine the significance $(*, \mathrm{P}<0.05)$. 

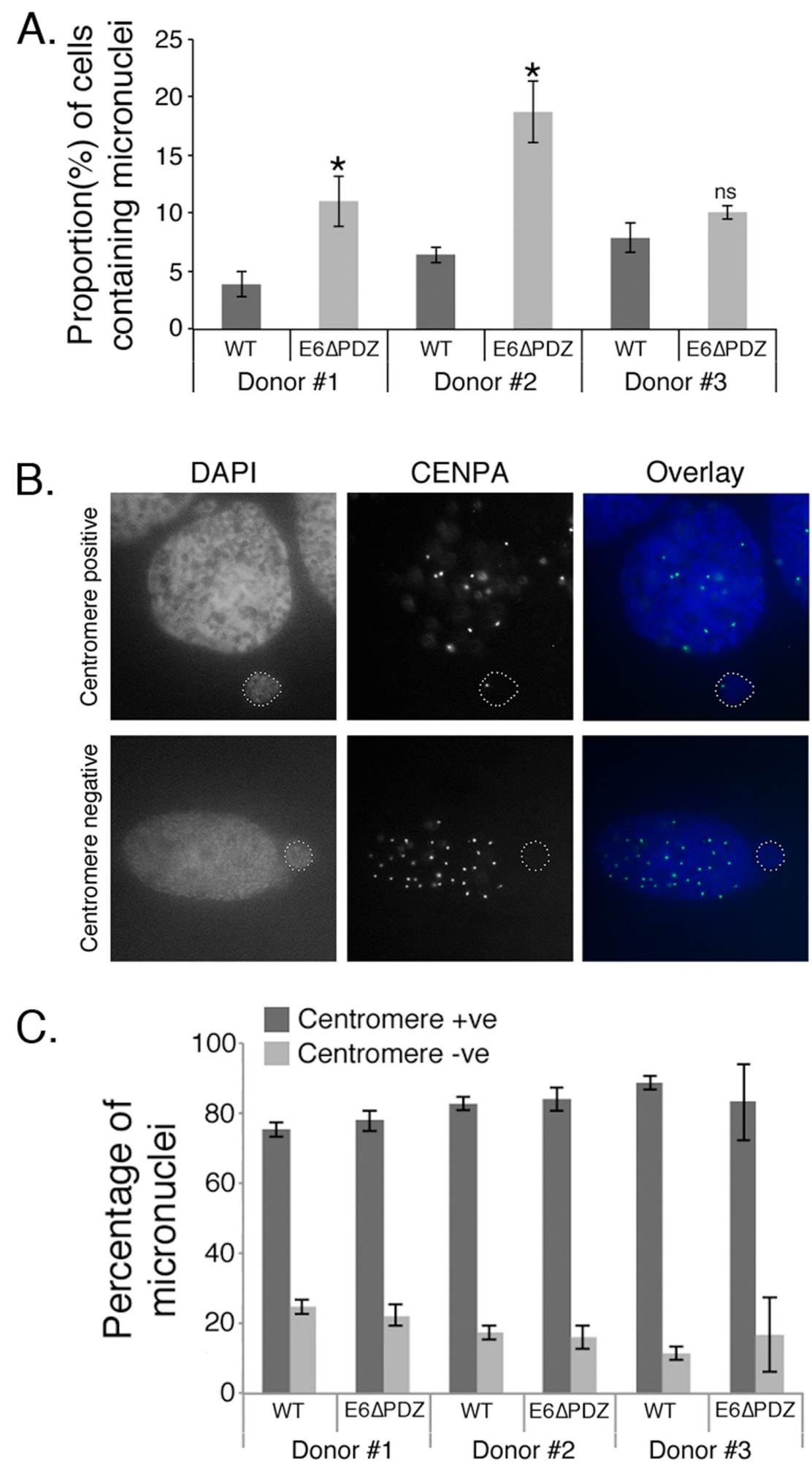

Figure 4: Increased micronuclei formation associated with E6 $\Delta \mathrm{PDZ}$ genome-containing cells. A. The proportion of cells containing micronuclei was scored from DAPI-stained cells. The data were collected from three independent experiments, with a minimum of 200 cells for each donor scored on each occasion. The data are shown as the mean ( \pm standard error of the mean), and in all three donors the frequency was increased in E6 $\triangle \mathrm{PDZ}$ genome-containing cells $(*, \mathrm{p}<0.05)$; ns, not significant). B. Images of examples of CENPA positive and negative nuclei; in overlay CENPA (green), nuclei (blue). C. The proportion of cells with micronuclei containing centromeres was determined by immunofluorescence staining for CENPA. The data were collected from three independent experiments, with a minimum of 200 cells with micronuclei scored for each experiment, and shown as the mean ( \pm standard error of the mean). 
WT and E6 $\triangle \mathrm{PDZ}$ genome-containing cells the majority of micronuclei were CENPA-positive and this was consistent across the three different donor backgrounds, with no significant difference in the frequencies of the two populations between the different viral genomes (Figure 4C). These data indicate that the formation of micronuclei in WT or mutant genome-containing cells occurs through similar mechanisms, although this is enhanced in the absence of the E6 PBM function. Moreover, since the majority of micronuclei formed in HPV-containing cells did not contain acentric chromosomes, this suggests that these micronuclei arise from chromosome segregation errors, in support of our previous data (Figure 3).

\section{Increased aneuploidy of cells containing E6 $\triangle P D Z$ genomes}

Both numerical and structural chromosomal abnormalities are a frequent feature of HPV oncoprotein expressing cells; and the expression of both E6 and E7 proteins induce mitotic abnormalities in keratinocytes [31, 33]. If, as our data indicate, the loss of the PDZ binding function of E6 causes an increase in abnormal cells in anaphase and telophase, and elevated levels of micronuclei formation, then this would lead to an increase in the frequency of chromosomal abnormalities. To examine this hypothesis, interphase fluorescence in situ hybridization (FISH) using two chromosome-specific centromeric probes against chromosomes 10 and 12 was performed on cells harboring WT or mutant E6 $\triangle \mathrm{PDZ}$ genomes. For each cell line, three cell passages were investigated (approximately 16 - 24 population doublings between the first and third passages tested). Importantly, since extended cell culture of E6 $\triangle \mathrm{PDZ}$ cell lines often leads to loss of episomes and viral DNA integration, only cell passages prior to these viral genetic events (as determined by Southern analysis [27]) were subjected to FISH analysis.

Figure 5 shows the distribution of diploid and nondiploid chromosome copy number in interphase cells from the three cell passages of both WT and E6 $\triangle \mathrm{PDZ}$
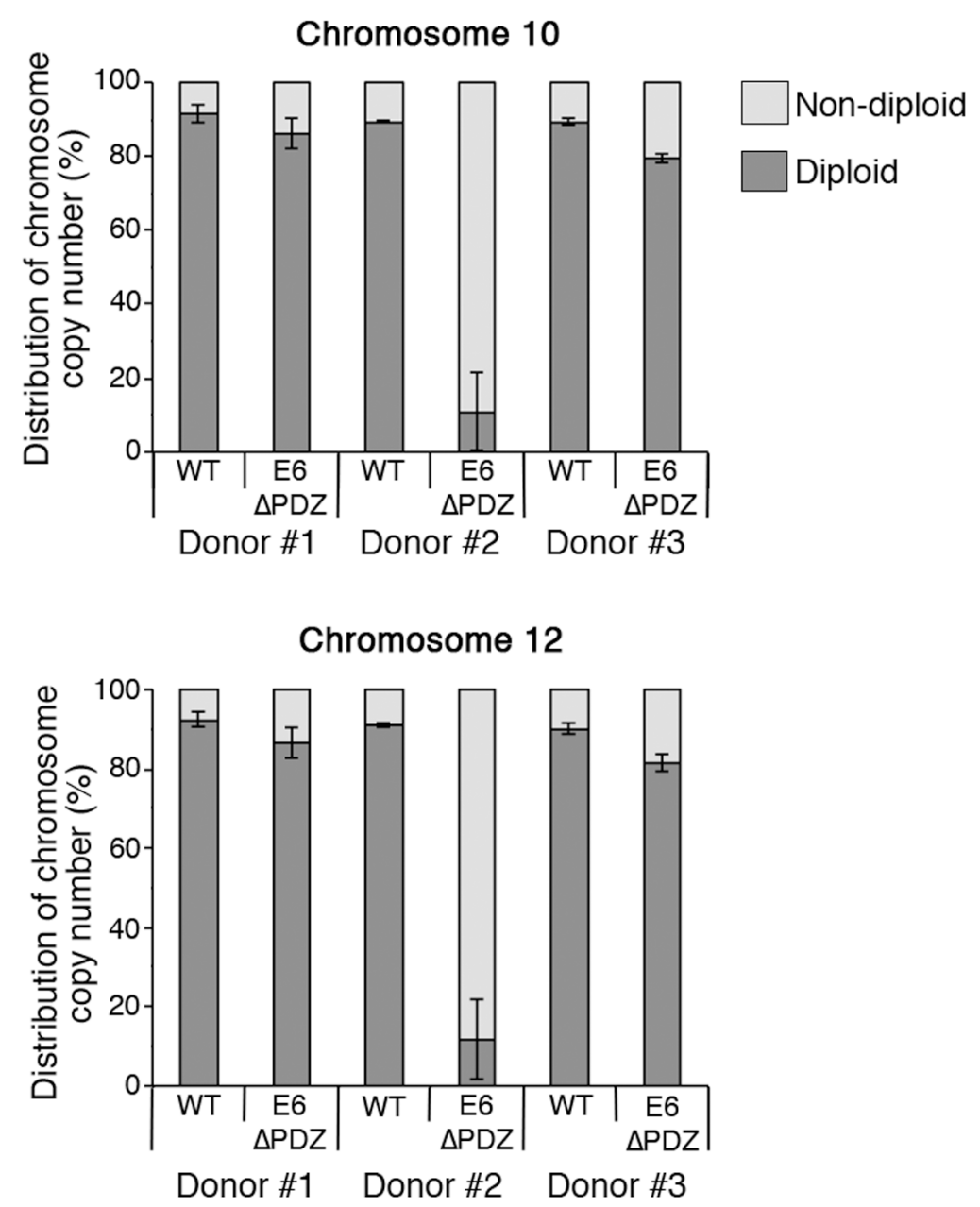

Figure 5: Increased aneuploidy of E6 $\triangle \mathrm{PDZ}$ genome-containing cells. Interphase fluorescence in situ hybridization using centromere enumeration probes for chromosomes 10 and 12. The data were collected from three different cell passages, with a minimum of 200 cells with single nuclei scored for each cell passage and given as the percentage of nuclei with diploid or non-diploid chromosome copy number (mean \pm standard error of the mean). The statistical significance of the increase in non-diploid copy number was: donor\#1, ns; donor\#2, $\mathrm{p}<0.01$ for chromosome 10 and 12; donor\#3, $\mathrm{p}<0.01$ for chromosome 10 and $\mathrm{p}<0.05$ for chromosome 12 . 
Table 1: Relative fold change in chromosome copy number in E6 $\Delta \mathrm{PDZ}$ genome-containing cells compared to cells harboring WT genomes

\begin{tabular}{lcccc}
\hline Donor & Chromosome & Copy number loss & Copy number gain & Copy number gain $>\mathbf{4}$ \\
\hline \multirow{2}{*}{$\mathbf{1}$} & 10 & 4.6 & 1.3 & 2.1 \\
& 12 & 0.7 & 2.0 & 2.6 \\
\# 2 & 10 & 0.9 & 9.8 & 24.3 \\
& 12 & 1.1 & 11.4 & 69.4 \\
\# & 10 & 1.6 & 1.9 & 6.5 \\
& 12 & 1.7 & 1.9 & 1.8 \\
\hline
\end{tabular}

genome-containing cells. In comparison to WT genome carrying cells, there was an increase in the frequency of E6 $\triangle \mathrm{PDZ}$ cells with a non-diploid genome content and this was observed across the three donor backgrounds (Figure $5)$. The level of increase in aneuploidy is statistically significant in two of the donors (donor $\# 2, \mathrm{p}<0.01$ for both chromosomes probes; donor \#3, $\mathrm{p}<0.01$ for chromosome 10 and $\mathrm{p}<0.05$ for chromosome 12). Whilst the frequency of E6 $\triangle \mathrm{PDZ}$ cells with non-diploid chromosome content is increased in all donors, it is markedly enhanced in one of the donors (donor \#2), by 8.4-fold (chromosome 10) and 10.0-fold (chromosome 12) compared to WT cells, suggesting donor-specific variation. There was no marked change in the frequency of cells with non-diploid chromosome copy number upon population-doubling of all the cell lines (i.e. comparison between the first and third cell passages, data not shown). Both loss and gain of chromosome copy number occurred in the presence of WT and E6 $\triangle \mathrm{PDZ}$ genomes. However, the prevailing trend across all three donors was increased frequency of cells with $>4$ chromosome copy number in cells carrying the mutant HPV18 genome defective in PDZ targeting (Table 1).

\section{Loss of E6-PDZ binding correlates with a change in the distribution of mitotic cells between the basal and suprabasal layers of organotypic raft cultures}

We have previously shown that upon organotypic raft growth, the stratified cultures containing the E6 $\triangle \mathrm{PDZ}$ mutant genome showed decreased hyperplastic morphology relative to the WT genomes; they do not show the characteristic thickening associated with replication of WT genomes and there was a reduction in expansion of $S$ phase-competent cells in the upper suprabasal layers [27]. Since our data thus far suggest that replication of the E6 $\triangle \mathrm{PDZ}$ mutant genomes is associated with mitotic abnormalities linked to spindle defects, we next wanted to determine if mitotic defects also occurred upon stratification of the E6 $\triangle \mathrm{PDZ}$ genome-containing cells, using $\alpha$-tubulin staining to identify mitotic cells in sections of the organotypic rafts.
In organotypic rafts of untransfected keratinocytes, few cells undergo mitosis and these are always restricted to the basal layers (Figure 6A). In contrast, mitotic cells showing $\alpha$-tubulin staining of the spindle were present in both the basal and suprabasal layers of rafts generated from the WT (Figure 6B, panels i, ii and iv) and the $\mathrm{E} 6 \triangle \mathrm{PDZ}$ (Figure $6 \mathrm{C}$, panels $\mathrm{i}$ - iii) genome-containing cells. However, the majority of mitotic WT cells were observed in the suprabasal compartment (basal, 20\%; suprabasal $80 \%$ ), consistent with the expansion of these epithelial layers (Figure 6D), whereas in rafts generated from cells harboring the E6 $\triangle \mathrm{PDZ}$ genomes, a significantly $(\mathrm{p}<0.01)$ greater proportion of mitotic cells remained in the basal layer compared to the WT raft cultures (basal, $71 \%$; suprabasal 29\%, Figure 6D). In the WT genome containing rafts, where polarity of the spindle was evident, it was most often bipolar (Figure 6B, panels i, ii and iv), although occasional cells with multipolar spindles were present (Figure 6B, panel iii). Interestingly, the polarity of the spindle could often not be determined in the E6 $\triangle \mathrm{PDZ}$ raft cultures, and where two poles were visible these were abnormal in appearance (Figure 6C, panel iii). Moreover, multipolar mitoses were present in E6 $\triangle \mathrm{PDZ}$ rafts (Figure $6 \mathrm{C}$, panels iv and v).

HR-HPV driven host genomic instability has been linked also to the induction of centrosome abnormalities by the viral oncoproteins $[34,35]$. Significantly, abnormal centrosome amplification is observed in stratified cultures of HPV16 episome-containing cells, indicating that this centrosome defect occurs during the normal replication cycle of the virus [30]. Thus, we sought to determine if centrosome amplification occurred in stratified cells harboring the HPV18 genomes by staining raft sections with $\gamma$-tubulin to visualize the centrosomes. The number of centrosomes in centrosome-positive cells was scored and cells with $>2$ centrosomes were quantified (Figure 6E). In the presence of WT genomes there was a significant increase in cells containing $>2$ centrosomes compared to the untransfected donor keratinocytes $(17.24 \%$ WT vs $8.79 \%$ HFK). Notably, these data concur with the findings derived from HPV16 genome-containing rafts [30]. A similar proportion of cells $(14.73 \%)$ with $>2$ centrosomes occurred in the stratified cultures of E6 $\triangle \mathrm{PDZ}$ genome- 

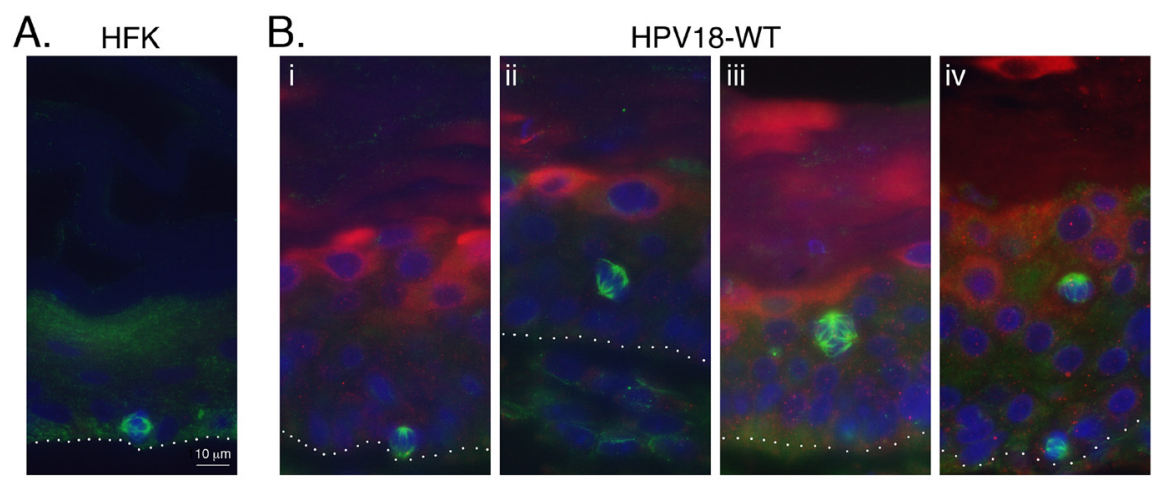

C.
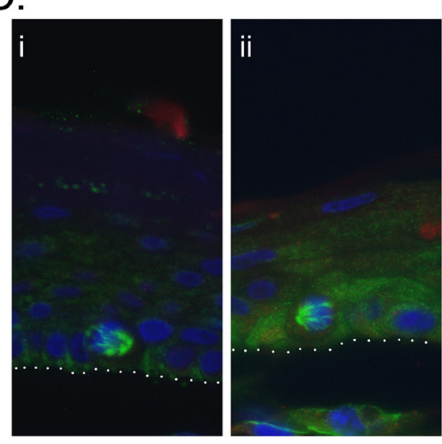

HPV18-E6 $\triangle \mathrm{PDZ}$


D.

Basal

Suprabasal

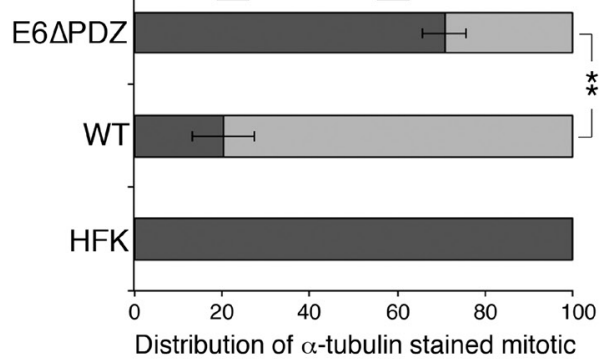

cells between basal and suprabasal layers (\%)

E.
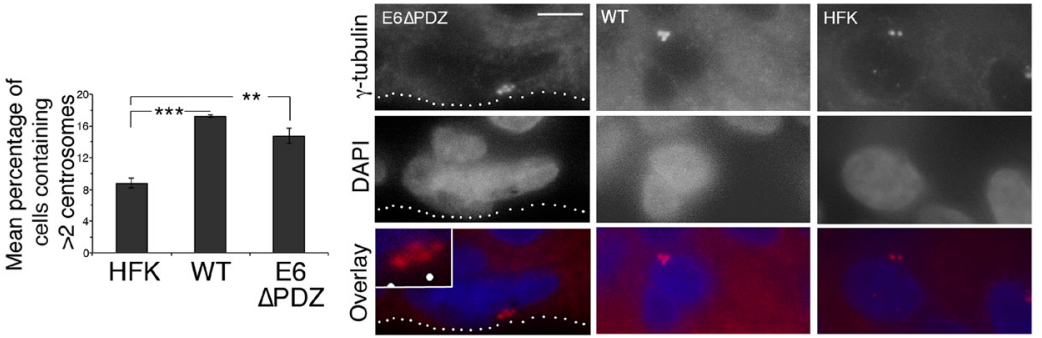

Figure 6: Loss of E6 PBM function is associated with an alteration in the distribution of $\alpha$-tubulin stained mitotic cells between basal and surpabasal cell layers of organotypic rafts. Sections of organotypic raft cultures of $\mathbf{A}$. untransfected HFK, B. HPV18 WT genome-containing cells and $\mathbf{C}$. cells containing E6 $\mathrm{PDDZ}$ genomes, were stained for $\alpha$-tubulin (green), HPV18 E4 (red), and DNA (blue). Examples given in B and C were taken from multiple donors. The dotted white line in A, B and C indicates the junction between the basal cell layer and fibroblast embedded collagen matrix. D. The distribution of mitotic cells between the basal and suprabasal cell layers of organotypic rafts shown as a percentage of total $\alpha$-tubulin stained mitotic cells ( \pm standard error of the mean). Data were collected from three donors, and for each donor five sections of organotypic raft were analyzed (full thickness and entire length). Numbers of $\alpha$-tubulin stained mitotic cells counted were 47 (HFK), 525 (WT) and 230 (E6 $\triangle \mathrm{PDZ}$ ). Student's $\mathrm{t}$ test was used to determine significance $(* *, p<0.01)$. E. Sections were stained with $\gamma$-tubulin to visualize centrosomes and cells with visible centrosomes were scored for centrosome number. Rafts prepared from three different donors were analyzed; numbers of cells scored were: 1772 (HFK), 4022 (WT) and 2758 (E6 $\triangle \mathrm{PDZ}$ ). Student's $\mathrm{t}$ test was used to determine significance $\left.{ }^{* *}, \mathrm{p}<0.01 ; * * *, \mathrm{p}<0.001\right)$. Shown are examples of cells in E6 $\triangle \mathrm{PDZ}$, WT rafts with $>2$ centrosomes, and an HFK raft with 2 centrosomes (red, $\gamma$-tubulin; blue, DAPI). The E6PDZ centrosomes are shown enlarged in the inset and the dotted white line indicates the junction between the basal cell layer and fibroblast embedded collagen matrix. Scale, $10 \mu \mathrm{m}$ (inset $18.5 \mu \mathrm{m}$ ). 
containing cells (Figure 6E). Thus, the exacerbation of mitotic dysregulation that we observe in HPV18 genomecontaining cells upon loss of the E6 PDZ binding function is independent of the virus-induced effects upon the centrosomes.

\section{DISCUSSION}

Our study has investigated the role of the E6 PBM in a physiologically significant HPV life cycle model based on primary human epidermal keratinocytes harboring episomal forms of the HPV18 genome [27, 36]. We have shown that a population of the keratinocytes harbouring the HR-HPV episomes undergo abnormal mitosis and that the size of this population increased upon loss of the four amino acids that constitute the HPV18 E6 PBM. In the presence of the WT genomes, the majority of cells that undergo abnormal mitosis exhibited metaphase associated spindle defects, as determined by $\alpha$-tubulin-staining, and very few cells with spindle-associated abnormalities were found to be in the mitotic late phases of anaphase or telophase. This suggests that in viral episome-containing cells, only a minor proportion of cells with metaphase spindle deformity can bypass the metaphase - anaphase checkpoint and thus exit into anaphase and telophase. These data are consistent with findings in cells expressing only the HPV16 E6 and E7 oncoproteins, where both E6 and E7 functions contribute to the induction of mitotic spindle abnormalities, including multipolar spindles, chromosome misalignment and lagging chromosomes [31]. The defects described are associated with inactivation of the mitotic spindle assembly checkpoint that ensures engagement of all kinetochores with the spindle prior to progression through to anaphase. The role of HPV16 E6 in the modulation of this checkpoint has been studied and associated with both p53-dependent and -independent pathways [32, 33, 37]. Notably, our data indicate that slippage through the metaphase-anaphase checkpoint is enhanced in the absence of the E6 PBM since a greater proportion of cells with abnormal spindle-associated defects are in anaphase and telophase. Thus, these data offer an explanation of the p53-independent effects previously observed.

It is well established that the E6 oncoprotein can disturb mitotic progression by interfering with multiple mitotic checkpoints [38]. The multinucleation of human keratinocytes co-expressing HPV16 E6 and E7 has been attributed to E6 oncoprotein function [35, 39], in complete agreement with our findings. Failure to protect E6expressing cells from defective cytokinesis and therefore from becoming multinucleate is linked to p53 inactivation $[34,39]$, but our data show that loss of the E6 PBM further perturbs mitotic division of episome-containing cells. In addition to causing an increase in the frequency of multinucleate cells, loss of the E6 PBM function also induces an increase in the degree of multinucleation of the cells, suggesting that the viability of multinucleate cells containing E6 $\triangle \mathrm{PDZ}$ genomes is greater than that of similar cells harboring WT genomes [35].

Our findings raise the interesting notion that the function of the E6 PBM of HPV18 acts to safeguard mitotic integrity in HPV-infected cells. Preserving mitotic integrity of viral genome-containing cells would be necessary to generate viable progeny, in which viral genomes can stably segregate between daughter cells and be maintained, as well as to support viral replication and promote persistence of episomes. This hypothesis would be consistent with our previous data demonstrating that HPV18 E6 $\triangle$ PDZ genome-containing cells lose episomes after extended cell passage, and are unable to support efficient viral genome establishment or viral DNA amplification upon stratification [27]. Moreover, loss of episomal maintenance also occurs in keratinocytes harboring HPV16 and 31 genomes that express a mutant E6 protein unable to target PDZ proteins [28, 40]. Interestingly, it has recently been shown that maintenance replication of the mutant HPV16 E6 $\triangle \mathrm{PDZ}$ genomes could be partially restored in immortalized keratinocytes by repression of $\mathrm{p} 53$ protein expression [41]. The mechanism was not disclosed, but the authors hypothesized that the E6 PBM promotes viral genome maintenance by neutralization of a p53 activity that is resistant to E6mediated degradation. These findings imply that the balance between E6 functions in infected cells is important for episomal maintenance and future studies should be directed to understanding the p53-PDZ protein axis in the HR-HPV life cycle. Moreover, a balance between E6 and E7 functions has also been shown to be important for episomal maintenance in primary keratinocytes [42]. Therefore, it cannot be ruled out that the role of the E6 PBM might also function to mediate interactions with other HPV proteins, either directly or indirectly.

Disentangling the E6 PBM function in terms of specific PDZ targets is complicated by the very large number of PDZ proteins that have been identified to interact with E6 via the PBM [43, 44]. Many of these PDZ substrates are degraded by E6 via the proteasome, or have altered cellular localization in HPV positive cervical cancer cells. However, our own analyses have not identified similar effects on specific E6 PDZ substrates in the context of cells harboring complete HR-HPV genomes [45], and similar observations have been made in overexpression studies (either E6 expressed alone or together with E7) in epidermal keratinocytes of transgenic mice or in primary foreskin keratinocytes in cell culture $[46,47]$. Furthermore, the interaction between E6 and PDZ proteins is most likely tightly regulated during the virus life cycle by phosphorylation of the E6 motif [18, 27]. Phosphorylation of the PBM blocks the interaction with PDZ proteins and enables E6 interaction with isoforms of the 14-3-3 proteins [18, 19, 44]. The E6 $\triangle \mathrm{PDZ}$ mutant is unable to interact with PDZ or 14-3-3 proteins. 
However, cells harboring a HPV18 mutant genome containing an E6 mutation that abrogates phosphorylation of the E6 PBM, thus blocking interaction with 14-33 isoforms, but confers constitutive targeting of PDZ proteins, retain stable replication of the viral episomes and support vegetative genome amplification [27]. Thus, this strongly indicates that the underlying mechanism of the E6 mitotic function described here involves targeting of PDZ proteins. Nevertheless, it remains to be determined what specific PDZ protein(s) may function to maintain mitotic and genomic stability.

In normal epithelia, asymmetric cell division of the basal cells is required for normal differentiation; producing two daughter cells with dissimilar fates of proliferation and differentiation. This process can become uncoupled, as in the developing mouse epidermis, where differentiating suprabasal daughter cells from basal mitotic divisions retain mitotic potential in order to rapidly form the multilayered epidermis [48]. The orientation of the mitotic spindle has an important role to play in the symmetry of cell division; the polarity complexes Par and Scribble that contain several of the E6 PBM substrates, including DLG1 and hScrib, and the 14-3-3 proteins, have important roles in directing the positioning of the mitotic spindle in Drosophila epithelial cells [49-51]. Moreover, some of the products of the mammalian homologues of these genes, including the PDZ protein PAR3, are also involved in directing spindle orientation in the developing embryonic mouse epidermis [48]. Notably, disturbance of mitotic spindle polarity in the mouse epidermis led to epidermal hyperproliferation and suprabasal mitoses $[48,52]$. In our previous study, we showed that disruption of the E6 PBM was linked to a reduction in the expansion of replication competent cells in the upper layers of organotypic rafts [27]. In support of this, we show here that whilst in the WT cells the majority of mitotic cells reside in the suprabasal layers, the converse occurs in the mutant E6 $\triangle \mathrm{PDZ}$ rafts, where the basal layer contains the majority of mitotic cells. Moreover, our data demonstrate that loss of the E6 PBM may perturb orientation of the mitotic spindle on organotypic raft culture, and leads to spindle defects in anaphase and telophase. One explanation for this is that in infected epithelia, HPV disturbs the polarity of mitotic cells to allow rapid suprabasal expansion, and the E6 PBM somehow contributes to the deregulation of pathways that control this polarity. Interestingly, E7 associates with the spindle protein nuclear mitotic apparatus protein [53], which is important for spindle positioning in asymmetric cell divisions [48], suggesting that both E6 and E7 may cooperate to alter mitotic cell polarity and this is necessary for viral persistence and vegetative replication.

The nature of the E6 PBM interaction with PDZ domains makes it an amenable peptide-protein interaction for inhibition by small molecules with the intention to develop novel therapeutics for the treatment of HPVassociated cancers $[54,55]$. Since the E6 PBM is essential for viral replication [27], inhibition of this function may be considered a route of anti-viral intervention to treat active infections. However, the data presented here offers a note of caution to disturbing the E6 PBM function in viral genome-containing cells. Firstly, loss of this function in this context is associated with viral DNA integration $[28,29]$, and this is a known risk factor for progression of HR-HPV infections to malignancy. In addition, we have demonstrated that viral episome-containing cells that have lost the E6 PBM function show increased genome instability in comparison to cells carrying the WT genomes. Thus, targeting this virus-host interaction therapeutically may instead accelerate the progression of HPV infections to malignancy. Also, it is worth noting that modulation of the PKA or AKT signaling pathways that lead to phosphorylation of the E6 PBM could be an independent risk factor for malignant progression by blocking E6 PDZ interactions [19, 27, 56].

Finally, this study suggests that one role of the E6 PBM in the HPV life cycle is to maintain mitotic viability of episome-containing cells, ultimately protecting the cells and permitting viral persistence. However, this E6 motif also prevents the cells from acquiring host genome instability and aneuploidy, both of which are associated with oncogenicity. Since the E6 PBM has been associated with acquisition of malignant phenotypes associated with the late stage of carcinogenesis [13, 14], this E6 function therefore has both tumor suppressor and oncogenic roles, dependent on the cellular context.

\section{MATERIALS AND METHODS}

\section{Cell culture and generation of HPV18 genome- containing cells}

Primary human foreskin keratinocytes (HFK) were isolated from new-born foreskin circumcision tissue samples collected from patients attending a general practitioner's practice, with informed written parental consent (Southampton and South West Hampshire research ethics committee A approval no. 06/Q1702/45). The cells were maintained in serum free media (SFM keratinocyte growth media, Life Technologies, Paisley, United Kingdom) as previously described [57]. Low passage fibroblasts (J2-3T3) were maintained in Dulbecco's modified media supplemented with $10 \%$ bovine serum (Gibco, Thermo Fisher Scientific, Cramlington, United Kingdom) and $4 \mathrm{mM}$ L-glutamine.

HFK from three different donors $(\# 1, \# 2, \# 3)$ were each transfected with the recircularized wild type (WT) and mutant (E6 $\triangle \mathrm{PDZ})$ HPV18 genomes and episomecontaining cell lines established, as described previously $[27,36]$. Briefly, following transfection, cells were plated onto a feeder layer of $\gamma$-irradiated J2-3T3 fibroblasts (3,000 rads) in E media containing fetal calf serum (HyClone, Thermo Fischer Scientific), epidermal growth 
factor (BD Biosciences, Oxford, United Kingdom) and G418 antibiotic (PAA laboratories, Yeovil, UK) [36]. The cells were maintained under drug selection for 8 days and henceforth grown in E medium without G418 antibiotic. Once cell colonies emerged, they were pooled and expanded on a fibroblast feeder layer. Presence of viral episomes in the HPV18 genome transfected cells lines was confirmed by Southern analysis of total DNA extracted from the monolayer grown cells using a $\left[\alpha-{ }^{32} \mathrm{P}\right]$ dCTP-labelled HPV18 genomic probe as described previously [36].

\section{Live cell microscopy}

Real-time images of cell growth on plastic were captured by a Cell-IQ system (CM Technologies) running Imagen software version 2.8.12.0 and analyzer version 3.3.0. Cells were seeded at a density of $2 \times 10^{4}$ cells onto a feeder layer of $\gamma$-irradiated J2-3T3 fibroblast cells in a twelve-well plate, and grown in complete E media (replaced every two days), until cell colonies were visible. The plate was then inserted into the Cell-IQ, and between three and five locations within each well were monitored every $7 \mathrm{~min}$ over a $16 \mathrm{~h}$ period at $10 \mathrm{x}$ magnification, in $5 \% \mathrm{CO}_{2}$ at $37^{\circ} \mathrm{C}$.

Mitotic events were scored from the resulting timelapse videos as 'normal' (based upon: bipolar, visible and equal separation and timing of mitosis) or otherwise 'abnormal'. For each cell line, between 14 and 23 colonies were counted, with every mitotic event within that colony scored, equating to 90 to 308 mitoses per cell line.

\section{Organotypic raft culture}

Organotypic raft cultures were grown at the air-liquid interface for 13 days and fixed with 3.7\% formaldehyde, as described previously [58]. Rafts were paraffin-embedded and $4 \mu \mathrm{m}$ sections prepared (Propath UK, Ltd., Hereford, United Kingdom).

\section{Phase contrast and immunofluorescence microscopy}

Keratinocytes were grown on $\gamma$-irradiated J2-3T3 cells seeded into wells of a six multi-well plate and viewed by phase contrast on Axiovert 100 microscope (Zeiss). For immunofluorescence microscopy, cells were grown on $\gamma$-irradiated J2-3T3 cells seeded onto glass coverslips. Once the keratinocytes had reached $60 \%$ confluence, the feeder cells were removed with EDTA in phosphate-buffered saline (PBS) and the cells were fixed in 3.6\% formaldehyde (EMGrade, TAAB laboratories, Aldermaston, Berks, United Kingdom) for $20 \mathrm{~min}$ and then permeabilized using $0.1 \%$ Triton X-100 for $5 \mathrm{~min}$. Fixed cells were pretreated with blocking buffer $(20 \% \mathrm{v} / \mathrm{v}$ heat-inactivated normal goat serum, $0.1 \% \mathrm{w} / \mathrm{v}$ bovine serum albumin in PBS) prior to addition of anti- $\alpha$-tubulin antibody at 1 in 2000 dilution (clone B512, Sigma-Aldrich) or anti-CENPA antibody at 1 in 500 dilution (3-19, Abcam, Cambridge, United Kingdom). Immune complexes were recognized by an anti-mouse Alexa 488 conjugate (Molecular Probes, Life Technologies). For staining of organotypic rafts, sections $(4 \mu \mathrm{m})$ were first de-paraffinized and subjected to low-temperature antigen retrieval as described by us previously [59]. Sections were then incubated with blocking buffer followed by staining with antibodies to $\alpha$-tubulin and HPV18 E4 antibody R424 [60], and $\gamma$-tubulin at 1 in 500 dilution (mouse monoclonal antibody, Abcam), and immune complexes detected using anti-mouse Alexa 488 or 594, and anti-rabbit Alexa 594 conjugates. Nuclei were counterstained with 4', 6'-diamidino-2-phenylindole (DAPI), and cells and raft sections mounted in ProLong ${ }^{\circledR}$ Gold Antifade reagent (Life Technologies). Stained cells and sections were viewed on a Nikon Eclipse E600 microscope fitted with epifluorescence.

\section{Interphase fluorescence in situ hybridization}

Cell suspensions were harvested after $15 \mathrm{~min}$ treatment with hypotonic solution $(0.075 \mathrm{M} \mathrm{KCl})$ prior to methanol:acetic acid $(3: 1 \mathrm{v} / \mathrm{v})$ fixation and then the cells were dropped onto clean glass slides. CEP10 and CEP12 probes (Cytocell, Cambridge, United Kingdom) were applied to the slides under a coverslip and hybridized overnight on a HYBrite (Abbott Molecular, Maidenhaed, United Kingdom). Slides were then washed in a solution containing the DNA stain DAPI (Cytocell, Cambridge, United Kingdom) and for each cell passage, at least 200 cells with single nuclei visualized.

\section{ACKNOWLEDGMENTS}

We are most grateful to Joseph Spitzer and his young patients for the collection and donation of foreskin tissue for the isolation of primary keratinocytes. We thank Margaret Hartley for excellent technical assistance.

\section{CONFLICTS OF INTEREST}

The authors disclose no potential conflicts of interest.

\section{GRANT SUPPORT}

This work was supported by grants from the Wellcome Trust $(093450 / \mathrm{Z} / 10 / \mathrm{Z}$ and 09345/B/10/Z) and Cancer Research UK (C427/A8049). LB acknowledges support from the Associazione Italiana per la Ricerca sul Cancro.

\section{REFERENCES}

1. Bouvard V, Baan R, Straif K, Grosse Y, Secretan B, El Ghissassi F, Benbrahim-Tallaa L, Guha N, Freeman C, 
Galichet L, Cogliano V. A review of human carcinogensPart B: biological agents. Lancet Oncol. 2009; 10:321-2.

2. de Sanjose S, Quint WG, Alemany L, Geraets DT, Klaustermeier JE, Lloveras B, Tous S, Felix A, Bravo LE, Shin HR, Vallejos CS, de Ruiz PA, Lima MA, et al. Human papillomavirus genotype attribution in invasive cervical cancer: a retrospective cross-sectional worldwide study. Lancet Oncol. 2010; 11:1048-56. doi: 10.1016/ S1470-2045(10)70230-8.

3. de Martel C, Ferlay J, Franceschi S, Vignat J, Bray F, Forman D, Plummer M. Global burden of cancers attributable to infections in 2008: a review and synthetic analysis. The Lancet Oncology. 2012; 13:607-15. doi: 10.1016/S1470-2045(12)70137-7.

4. St Guily JL, Clavel C, Okais C, Pretet JL, Beby-Defaux A, Agius G, Birembaut P, Jacquard AC, Leocmach Y, Soubeyrand B, Riethmuller D, Denis F, Mougin C. Human papillomavirus genotype distribution in tonsil cancers. Head \& neck oncology. 2011; 3:6. doi: 10.1186/1758-3284-3-6.

5. Moody CA, Laimins LA. Human papillomavirus oncoproteins: pathways to transformation. Nature reviews Cancer. 2010; 10:550-60. doi: 10.1038/nrc2886.

6. Banks L, Pim D, Thomas M. Human tumour viruses and the deregulation of cell polarity in cancer. Nature reviews Cancer. 2012; 12:877-86. doi: 10.1038/nrc3400.

7. Kiyono T, Hiraiwa A, Fujita M, Hayashi Y, Akiyama T, Ishibashi M. Binding of high-risk human papillomavirus E6 oncoproteins to the human homologue of the Drosophila discs large tumor suppressor protein. Proceedings of the National Academy of Sciences of the United States of America. 1997; 94:11612-6.

8. Shai A, Brake T, Somoza C, Lambert PF. The human papillomavirus E6 oncogene dysregulates the cell cycle and contributes to cervical carcinogenesis through two independent activities. Cancer research. 2007; 67:1626-35. doi: 10.1158/0008-5472.CAN-06-3344.

9. Nguyen ML, Nguyen MM, Lee D, Griep AE, Lambert PF. The PDZ ligand domain of the human papillomavirus type 16 E6 protein is required for E6's induction of epithelial hyperplasia in vivo. Journal of virology. 2003; 77:6957-64.

10. Nguyen MM, Nguyen ML, Caruana G, Bernstein A, Lambert PF, Griep AE. Requirement of PDZ-containing proteins for cell cycle regulation and differentiation in the mouse lens epithelium. Molecular and cellular biology. 2003; 23:8970-81.

11. Spanos WC, Geiger J, Anderson ME, Harris GF, Bossler AD, Smith RB, Klingelhutz AJ, Lee JH. Deletion of the PDZ motif of HPV16 E6 preventing immortalization and anchorage-independent growth in human tonsil epithelial cells. Head \& neck. 2008; 30:139-47. doi: 10.1002/ hed.20673.

12. Muench P, Hiller T, Probst S, Florea AM, Stubenrauch F, Iftner T. Binding of PDZ proteins to HPV E6 proteins does neither correlate with epidemiological risk classification nor with the immortalization of foreskin keratinocytes. Virology. 2009; 387:380-7. doi: 10.1016/j.virol.2009.02.018.

13. Spanos WC, Hoover A, Harris GF, Wu S, Strand GL, Anderson ME, Klingelhutz AJ, Hendriks W, Bossler AD, Lee JH. The PDZ binding motif of human papillomavirus type 16 E6 induces PTPN13 loss, which allows anchorageindependent growth and synergizes with ras for invasive growth. J Virol. 2008; 82:2493-500. doi: 10.1128/ JVI.02188-07.

14. Watson RA, Thomas M, Banks L, Roberts S. Activity of the human papillomavirus E6 PDZ-binding motif correlates with an enhanced morphological transformation of immortalized human keratinocytes. Journal of cell science. 2003; 116:4925-34. doi: 10.1242/jcs.00809.

15. Gardiol D, Kuhne C, Glaunsinger B, Lee SS, Javier R, Banks L. Oncogenic human papillomavirus E6 proteins target the discs large tumour suppressor for proteasomemediated degradation. Oncogene. 1999; 18:5487-96. doi: 10.1038/sj.onc. 1202920.

16. Facciuto F, Bugnon Valdano M, Marziali F, Massimi P, Banks L, Cavatorta AL, Gardiol D. Human papillomavirus (HPV)-18 E6 oncoprotein interferes with the epithelial cell polarity Par3 protein. Molecular oncology. 2014; 8:533-43. doi: 10.1016/j.molonc.2014.01.002.

17. Hampson L, Li C, Oliver AW, Kitchener HC, Hampson IN. The PDZ protein Tip-1 is a gain of function target of the HPV16 E6 oncoprotein. Int J Oncol. 2004; 25:1249-56.

18. Kuhne C, Gardiol D, Guarnaccia C, Amenitsch H, Banks L. Differential regulation of human papillomavirus E6 by protein kinase A: conditional degradation of human discs large protein by oncogenic E6. Oncogene. 2000; 19:5884-91.

19. Boon SS, Banks L. High-risk human papillomavirus E6 oncoproteins interact with 14-3-3 zeta in a PDZ binding motif-dependent manner. Journal of virology. 2013; 87:1586-95. doi: 10.1128/JVI.02074-12.

20. Storrs $\mathrm{CH}$, Silverstein SJ. PATJ, a tight junction-associated PDZ protein, is a novel degradation target of high-risk human papillomavirus E6 and the alternatively spliced isoform 18 E6. Journal of virology. 2007; 81:4080-90. doi: 10.1128/JVI.02545-06.

21. Nakagawa S, Huibregtse JM. Human scribble (Vartul) is targeted for ubiquitin-mediated degradation by the highrisk papillomavirus E6 proteins and the E6AP ubiquitinprotein ligase. Molecular and cellular biology. 2000; 20:8244-53.

22. Lee SS, Weiss RS, Javier RT. Binding of human virus oncoproteins to $\mathrm{hDlg} / \mathrm{SAP} 97$, a mammalian homolog of the Drosophila discs large tumor suppressor protein. Proceedings of the National Academy of Sciences of the United States of America. 1997; 94:6670-5.

23. Thomas M, Laura R, Hepner K, Guccione E, Sawyers C, Lasky L, Banks L. Oncogenic human papillomavirus E6 proteins target the MAGI-2 and MAGI-3 proteins for 
degradation. Oncogene. 2002; 21:5088-96. doi: 10.1038/ sj.onc. 1205668.

24. Glaunsinger BA, Lee SS, Thomas M, Banks L, Javier R. Interactions of the PDZ-protein MAGI-1 with adenovirus E4-ORF1 and high-risk papillomavirus E6 oncoproteins. Oncogene. 2000; 19:5270-80. doi: 10.1038/sj.onc.1203906.

25. Spanos WC, Hoover A, Harris GF, Wu S, Strand GL, Anderson ME, Klingelhutz AJ, Hendriks W, Bossler AD, Lee JH. The PDZ binding motif of human papillomavirus type 16 E6 induces PTPN13 loss, which allows anchorageindependent growth and synergizes with ras for invasive growth. Journal of virology. 2008; 82:2493-500. doi: 10.1128/JVI.02188-07.

26. Jing $\mathrm{M}$, Bohl J, Brimer N, Kinter M, Vande Pol SB. Degradation of tyrosine phosphatase PTPN3 (PTPH1) by association with oncogenic human papillomavirus E6 proteins. Journal of virology. 2007; 81:2231-9. doi: 10.1128/JVI.01979-06.

27. Delury CP, Marsh EK, James CD, Boon SS, Banks L, Knight GL, Roberts S. The role of protein kinase A regulation of the E6 PDZ binding domain during differentiation dependent life cycle of human papillomavirus type 18. Journal of virology. 2013; 87:9463-72. doi: 10.1128/JVI.01234-13.

28. Nicolaides L, Davy C, Raj K, Kranjec C, Banks L, Doorbar J. Stabilization of HPV16 E6 protein by PDZ proteins, and potential implications for genome maintenance. Virology. 2011; 414:137-45. doi: 10.1016/j.virol.2011.03.017.

29. Lee C, Laimins LA. Role of the PDZ domain-binding motif of the oncoprotein E6 in the pathogenesis of human papillomavirus type 31. J Virol. 2004; 78:12366-77. doi: 10.1128/JVI.78.22.12366-12377.2004.

30. Duensing S, Duensing A, Flores ER, Do A, Lambert $\mathrm{PF}$, Munger K. Centrosome abnormalities and genomic instability by episomal expression of human papillomavirus type 16 in raft cultures of human keratinocytes. Journal of virology. 2001; 75:7712-6. doi: 10.1128/ JVI.75.16.7712-7716.2001.

31. Duensing S, Munger K. The human papillomavirus type 16 E6 and E7 oncoproteins independently induce numerical and structural chromosome instability. Cancer research. 2002; 62:7075-82.

32. Thomas JT, Laimins LA. Human papillomavirus oncoproteins E6 and E7 independently abrogate the mitotic spindle checkpoint. J Virol. 1998; 72:1131-7.

33. Plug-Demaggio AW, McDougall JK. The human papillomavirus type $16 \mathrm{E} 6$ oncogene induces premature mitotic chromosome segregation. Oncogene. 2002; 21:7507-13. doi: 10.1038/sj.onc.1205903.

34. Duensing S, Lee LY, Duensing A, Basile J, Piboonniyom $\mathrm{S}$, Gonzalez S, Crum CP, Munger $\mathrm{K}$. The human papillomavirus type $16 \mathrm{E} 6$ and $\mathrm{E} 7$ oncoproteins cooperate to induce mitotic defects and genomic instability by uncoupling centrosome duplication from the cell division cycle. Proceedings of the National Academy of Sciences of the United States of America. 2000; 97:10002-7. doi: 10.1073/pnas.170093297.

35. Duensing S, Duensing A, Crum CP, Munger K. Human papillomavirus type $16 \mathrm{E} 7$ oncoprotein-induced abnormal centrosome synthesis is an early event in the evolving malignant phenotype. Cancer research. 2001; 61:2356-60.

36. Wilson R, Ryan GB, Knight GL, Laimins LA, Roberts S. The full-length E1E4 protein of human papillomavirus type 18 modulates differentiation-dependent viral DNA amplification and late gene expression. Virology. 2007; 362:453-60. doi: 10.1016/j.virol.2007.01.005.

37. Patel D, McCance DJ. Compromised spindle assembly checkpoint due to altered expression of Ubch10 and Cdc20 in human papillomavirus type 16 E6- and E7-expressing keratinocytes. Journal of virology. 2010; 84:10956-64. doi: 10.1128/JVI.00259-10.

38. Thompson DA, Belinsky G, Chang TH, Jones DL, Schlegel R, Munger K. The human papillomavirus-16 E6 oncoprotein decreases the vigilance of mitotic checkpoints. Oncogene. 1997; 15:3025-35. doi: 10.1038/sj.onc.1201495.

39. Wallace NA, Robinson K, Galloway DA. Beta human papillomavirus E6 expression inhibits stabilization of p53 and increases tolerance of genomic instability. Journal of virology. 2014; 88:6112-27. doi: 10.1128/JVI.03808-13.

40. Lee C, Laimins LA. Role of the PDZ domain-binding motif of the oncoprotein E6 in the pathogenesis of human papillomavirus type 31. Journal of virology. 2004; 78:12366-77. doi: 10.1128/JVI.78.22.12366-12377.2004.

41. Brimer N, Vande Pol SB. Papillomavirus E6 PDZ interactions can be replaced by repression of $\mathrm{p} 53$ to promote episomal human papillomavirus genome maintenance. Journal of virology. 2014; 88:3027-30. doi: 10.1128/ JVI.02360-13.

42. Park RB, Androphy EJ. Genetic analysis of high-risk e6 in episomal maintenance of human papillomavirus genomes in primary human keratinocytes. J Virol. 2002; 76:11359-64.

43. Thomas M, Myers MP, Massimi P, Guarnaccia C, Banks L. Analysis of multiple HPV E6 PDZ interactions defines type specific PDZ fingerprints that predict oncogenic potential. PLoS Pathog. 2016; 12:e1005766. doi: 10.1371/journal. ppat.1005766.

44. James CD, Roberts S. Viral Interactions with PDZ DomainContaining Proteins-An Oncogenic Trait? Pathogens. 2016; 5. doi: 10.3390/pathogens5010008.

45. James CD. (2015). A study of PDZ protein expression during the human papillomavirus life cycle. $\mathrm{Ph}$. D. thesis. University of Birmingham, Birmingham, United Kingdom.

46. Simonson SJ, Difilippantonio MJ, Lambert PF. Two distinct activities contribute to human papillomavirus 16 E6's oncogenic potential. Cancer research. 2005; 65:8266-73. doi: 10.1158/0008-5472.CAN-05-1651.

47. Choi M, Lee S, Choi T, Lee C. Roles of the PDZ domainbinding motif of the human papillomavirus type 16 E6 on 
the immortalization and differentiation of primary human foreskin keratinocytes. Virus genes. 2014; 48:224-32. doi: 10.1007/s11262-013-1017-9.

48. Lechler T, Fuchs E. Asymmetric cell divisions promote stratification and differentiation of mammalian skin. Nature. 2005; 437:275-80. doi: 10.1038/nature03922.

49. Lu MS, Prehoda KE. A NudE/14-3-3 pathway coordinates dynein and the kinesin Khc73 to position the mitotic spindle. Developmental cell. 2013; 26:369-80. doi: 10.1016/j.devcel.2013.07.021.

50. Bergstralh DT, Lovegrove HE, St Johnston D. Discs large links spindle orientation to apical-basal polarity in Drosophila epithelia. Current biology: CB. 2013; 23:170712. doi: 10.1016/j.cub.2013.07.017.

51. Nakajima Y, Meyer EJ, Kroesen A, McKinney SA, Gibson MC. Epithelial junctions maintain tissue architecture by directing planar spindle orientation. Nature. 2013; 500:35962. doi: 10.1038/nature12335.

52. Vasioukhin V, Bauer C, Degenstein L, Wise B, Fuchs E. Hyperproliferation and defects in epithelial polarity upon conditional ablation of alpha-catenin in skin. Cell. 2001; 104:605-17.

53. Nguyen CL, Munger K. Human papillomavirus E7 protein deregulates mitosis via an association with nuclear mitotic apparatus protein 1. Journal of virology. 2009; 83:1700-7. doi: 10.1128/JVI.01971-08.

54. Ramirez J, Poirson J, Foltz C, Chebaro Y, Schrapp M, Meyer A, Bonetta A, Forster A, Jacob Y, Masson M, Deryckere F, Trave G. Targeting the two oncogenic functional sites of the HPV E6 oncoprotein with a highaffinity bivalent ligand. Angewandte Chemie. 2015. doi: 10.1002/anie.201502646.
55. Belyaeva TA, Nicol C, Cesur O, Trave G, Blair GE, Stonehouse NJ. An RNA aptamer targets the PDZ binding motif of the HPV16 E6 oncoprotein. Cancers. 2014; 6:155369. doi: 10.3390/cancers6031553.

56. Boon SS, Tomaic V, Thomas M, Roberts S, Banks L. Cancer-causing human papillomavirus E6 proteins display major differences in the phospho-regulation of their PDZ interactions. Journal of virology. 2015; 89:1579-86. doi: 10.1128/JVI.01961-14.

57. Wilson R, Laimins LA. Differentiation of HPV-containing cells using organotypic "raft" culture or methylcellulose. Methods in molecular medicine. 2005; 119:157-69. doi: 10.1385/1-59259-982-6:157.

58. Knight GL, Pugh AG, Yates E, Bell I, Wilson R, Moody CA, Laimins LA, Roberts S. A cyclin-binding motif in human papillomavirus type 18 (HPV18) E1-E4 is necessary for association with CDK-cyclin complexes and G2/M cell cycle arrest of keratinocytes, but is not required for differentiation-dependent viral genome amplification or L1 capsid protein expression. Virology. 2011; 412:196-210. doi: 10.1016/j.virol.2011.01.007.

59. Watson RA, Rollason TP, Reynolds GM, Murray PG, Banks L, Roberts S. Changes in expression of the human homologue of the Drosophila discs large tumour suppressor protein in high-grade premalignant cervical neoplasias. Carcinogenesis. 2002; 23:1791-6.

60. Roberts S, Hillman ML, Knight GL, Gallimore PH. The ND10 component promyelocytic leukemia protein relocates to human papillomavirus type $1 \mathrm{E} 4$ intranuclear inclusion bodies in cultured keratinocytes and in warts. Journal of virology. 2003; 77:673-84. 